\title{
Cost-effectiveness analysis of intraoperative radiation therapy versus external beam radiation therapy for the adjuvant treatment of early breast cancer: A systematic review
}

\author{
Mahmoud Eisavi ${ }^{1}$, Aziz Rezapour ${ }^{2}$, Vahid Alipour ${ }^{2}$, Hamid Reza Mirzaei ${ }^{3}$, Jalal Arabloo*2 (D) \\ Received: 6 Apr 2020 \\ Published: 10 Dec 2020
}

\section{Abstract}

Background: Breast-conserving surgery (BCS) is the recommended treatment for early breast cancer. After BCS. Whole-breast external beam radiotherapy (WB-EBRT) is the standard of care. A possible alternative to post-operative WB-EBRT is intraoperative radiation therapy (IORT). The objectives of this systematic review were to analyses the cost-effectiveness of IORT versus EBRT for early-stage breast cancer and to assess the reporting quality of the included studies to inform future studies.

Methods: A systematic literature search was carried out in five main databases (PubMed, Scopus, Embase, Cochrane library, and Web of Science) to identify original studies published to June 25, 2020. We included all full economic evaluation studies (cost-effectiveness analysis (CEA), cost-utility analysis (CUA), and cost-benefit analysis (CBA), Model-based or trial-based) that assessed and compared IORT and EBRT in patients with early operable breast cancer. Study outcomes included cost per life-years gained or cost per qualityadjusted life-years (QALYs) gained or in monetary units or incremental cost-effectiveness ratio (ICER). The quality of the included articles was assessed using the Consolidated Health Economic Evaluation Reporting Standards (CHEERS) checklist. This review has been conducted and reported in accordance with the Preferred Reporting Items for Systematic Reviews and Meta-Analyses (PRISMA) statement.

Results: Of 1155 studies identified, eight studies met the inclusion criteria. In four studies, IORT was associated with lower costs and higher effectiveness than EBRT. In three studies, the dominant option was EBRT. In these studies, IORT also had lower costs and lower effectiveness than EBRT. Existing evidence suggests that IORT can be a cost-effective alternative to early breast cancer treatment by reducing therapeutic costs. Variables of cost-effectiveness were treatment costs, health state utilities, local and distant recurrence rates, and the probabilities of metastasis after treatment, recurrent cancer and death for both IORT and EBRT. The reporting quality of the included studies was "high" in five, "medium quality" in one and "low" in two studies.

Conclusion: Current evidence is sparse, and the number of studies was small but this evidence proposes that IORT can be a potential cost-saving strategy to the health systems for the adjuvant treatment of early breast cancer if the technology was carried out routinely in eligible patients. However, these results should be interpreted with caution because of the heterogeneity of studies and possible publication bias.

Keywords: Systematic review, Breast cancer, Cost-effectiveness analysis, Intraoperative radiation therapy, External beam radiation therapy

\section{Conflicts of Interest: None declared \\ Funding: None}

\section{*This work has been published under CC BY-NC-SA 1.0 license. Copyright $\odot$ Iran University of Medical Sciences}

Cite this article as: Eisavi M, Rezapour A, Alipour V, Mirzaei HR, Arabloo J. Cost-effectiveness analysis of intraoperative radiation therapy versus external beam radiation therapy for the adjuvant treatment of early breast cancer: A systematic review. Med J Islam Repub Iran. 2020 (10 Dec);34:167. https://doi.org/10.47176/mjiri.34.167

Corresponding author: Dr Jalal Arabloo, arabloo.j@iums.ac.ir

1. Faculty of Economics, Allameh Tabataba'i University, Tehran, Iran

2. Health Management and Economics Research Center, Iran University of Medical Sciences, Tehran, Iran

3. Cancer Research Center, Shahid Beheshti University of Medical Sciences, Tehran, Iran $\uparrow$ What is "already known" in this topic:

New less invasive technologies such as intraoperative radiation therapy (IORT) can play an essential role for patients who cannot use external beam radiotherapy (EBRT).

$\rightarrow$ What this article adds:

IORT has the potential to provide comparable or better outcomes for patients for early breast cancer and to be a cost-effective strategy from the health system perspective compared with EBRT. 


\section{Introduction}

Breast cancer is the most common cancer and the leading cause of cancer deaths and disability-adjusted life-years (DALYs) among women worldwide (1) . For a large proportion of women with early localized breast cancer, the recommended treatment is breast-conserving surgery (BCS) followed by postoperative radiotherapy, wholebreast external-beam radiation therapy (EBRT), which requires daily therapy for 4-7 weeks (2-4). EBRT after lumpectomy reduces the risk of local recurrence more than $10 \%$ at 5 years and reduces the risk of breast cancer death at 15 years for women with early invasive breast cancer $(5,6)$. Thus, post-operative WB-EBRT is the standard of care for patients with early invasive breast cancer after breast-conserving surgery (7). However, EBRT has some disadvantages. The long course of treatment is uncomfortable for patients and may require several travels to receive care . WB-EBRT may also be associated with short and long term adverse effects and can be impossible to deliver effectively in all patients (8).

New less invasive technologies such as IORT can play an essential role for patients who cannot use EBRT . The large international multicenter randomized controlled trial (RCT) of targeted intraoperative radiotherapy-alone (TARGIT-A) has confirmed the safety and effectiveness of the technique of targeted intraoperative radiotherapy (TARGIT-IORT) in women with early breast cancer (9). TARGIT-IORT and EBRT resulted in similar local recurrence-free survival (10). IORT requires only 25-30 min for a single dose of radiation treatment, greatly reducing the time and travel costs required for whole breast radiation therapy. IORT could potentially improve access to breast conservation by reducing costs and time required for patients to receive radiotherapy in resource-limited settings. Therefore, it is an interesting alternative for women who are candidates for breast-conserving surgery (11).

Considering the resources limitations, along with the above-mentioned innovations in the management of breast cancer, providing a light picture of the economic aspects of the technologies is increasingly important to help policymakers to efficiently allocate health system resources. The purposes of this systematic review were to identify the relevant economic evaluation studies of intraoperative radiation therapy versus external beam radiation therapy, assess the quality of the included studies to support future costeffectiveness studies in this field, and summarize the costeffectiveness results on the existing therapies of early breast cancer.

\section{Methods}

\section{Identification of studies}

A systematic search was conducted in PubMed, Scopus, Embase, Cochrane library, and Web of Science to identify original studies published up to June 25, 2020. The search strategy consisted of keywords and Mesh. There was no restriction on language or publication date. Separate search strategies were developed for each database (Appendix 1). List of references of eligible full text articles were further screened in order to find eligible studies. Studies were required to meet the following criteria in order to be included in the review:

- Population: people with early operable breast cancer;

- Intervention: IORT with or without post-operative WB-EBRT;

- Comparator: WB-EBRT delivered by linear accelerator after BSC;

- Outcomes: cost per life-years gained or cost per quality-adjusted life-years (QALYs) gained or in monetary units or incremental cost-effectiveness ratio (ICER)];

- Study design: Full economic evaluation studies (costeffectiveness analysis (CEA), cost-utility analysis (CUA), and cost-benefit analysis (CBA), Model-based or trialbased;

- Setting, country: all countries, all settings

Exclusion criteria were:

- Partial economic evaluation studies (cost-minimization analysis, cost-analysis) or non-economic evaluation studies

- Reviews, Commentaries (letters to the editors, editorials), protocols, Abstracts or conference presentations

- Non-English language full-text studies

- Duplicated publications

\section{Selection of Studies}

After removing duplicates, titles and abstracts of studies were screened independently by two researchers to identify all studies that potentially met the inclusion/exclusion criteria detailed above. Fulltexts of selected studies that appeared potentially relevant were obtained. These were assessed by one researcher against the eligibility criteria and checked independently by a second researcher. Any disagreements were resolved by discussion. The agreement was reached on all included studies.

Data extraction and quality assessment of the studies

Data extraction was performed by one reviewer (JA) and checked by a second reviewer (VA). Disagreements were resolved by discussion at each stage. Data were extracted using a researcher-made extraction table. Data extracted from each study included publication year, country, perspective, willing-to-pay threshold, type of economic evaluation, modeling approach, model states, time horizon, discount rates (costs, QALY), type of costing, included costs, outcome measures, type of sensitivity analysis, industry funding, population, comparators, and results.

Included studies were then assessed using the CHEERS checklist $(12,13)$. The CHEERS tool consists of twentyfour items in six sections (title and abstract, introduction, methods, results, discussion, and other) and were scored using 'Yes' (reported in full), 'Partially reported', 'No' (not reported), and 'Not Applicable'. Two researchers (JA and VA) independently assessed the included studies with disagreements resolved through consensus. In order to estimate a score of reporting, we allocated a score of 1 for each item that was reported in full, 0.5 for a partial report and otherwise 0 . Therefore, the maximum score for each study was 24 (14). Then, the studies were classified based on 
quantitative CHEERS scores in three categories of "high quality" for scores over 75\%, "moderate quality" between 50 and $75 \%$ and "low quality" below 50\%.

\section{Data synthesis}

Data were synthesized qualitatively, with tabulation of the key characteristics and results of included studies. This systematic review has been conducted and reported in accordance with the Preferred Reporting Items for Systematic Reviews and Meta-Analyses (PRISMA) statement (15).

\section{Results}

\section{Study Selection Process}

The searches identified 1155 citations (Fig. 1). After the removal of duplicates and screening of title/abstract, 12 articles were eligible for full-text assessment. Four studies were excluded because the studies were published as abstracts (16-18) or had irrelevant outcomes (19). Finally, 8 studies were included in the systematic review.

\section{Overview of Included Studies}

The key characteristics of all included studies are reported in Table 1. All studies were published between 2013 and 2019. Four studies were conducted in the USA (20$23)$, three studies in the $\operatorname{UK}(8,24,25)$ and one study in Czech (26). All studies were used a Markov modeling approach, except the studies of Shah et al. (23) and Vaidya et al. (25) that used reimbursement models or were trialbased. Two studies were applied societal perspectives $(20$, $23)$, two used a payer perspective $(22,26)$, one study both societal and health care sector (21) and the remaining studies reported results from the perspective of the National Health Service (NHS) $(8,24,25)$. All studies used qualityadjusted life-years (QALYs) as the effectiveness outcome. One study applied a 5 years' time horizon (25), three studies a 10 years' time horizon $(20,23,24)$, two studies a 40 years' time horizon $(8,26)$ and two studies considered a lifetime horizon $(21,22)$. Sensitivity analysis was conducted in the majority of included studies $(\mathrm{N}=7)$ although the type of approaches varied $(8,20-22,24-26)$.

\section{Quality of Reporting Assessment}

The summary results of the quality of reporting assessment for each study are presented in Table 2. Figure 2 illustrates the proportion of studies reported 'in full', 'Partially', 'not reported' and 'Not Applicable'. Quality scores ranged from 7 to 23 out of a maximum value of 24 points, with an average score of 17 (Table 2). Five studies were classified in the category of "high quality" $(8,22,24,25)$ one into (20) the "medium quality" category and two studies $(23,26)$ fell into the "low" reporting quality.

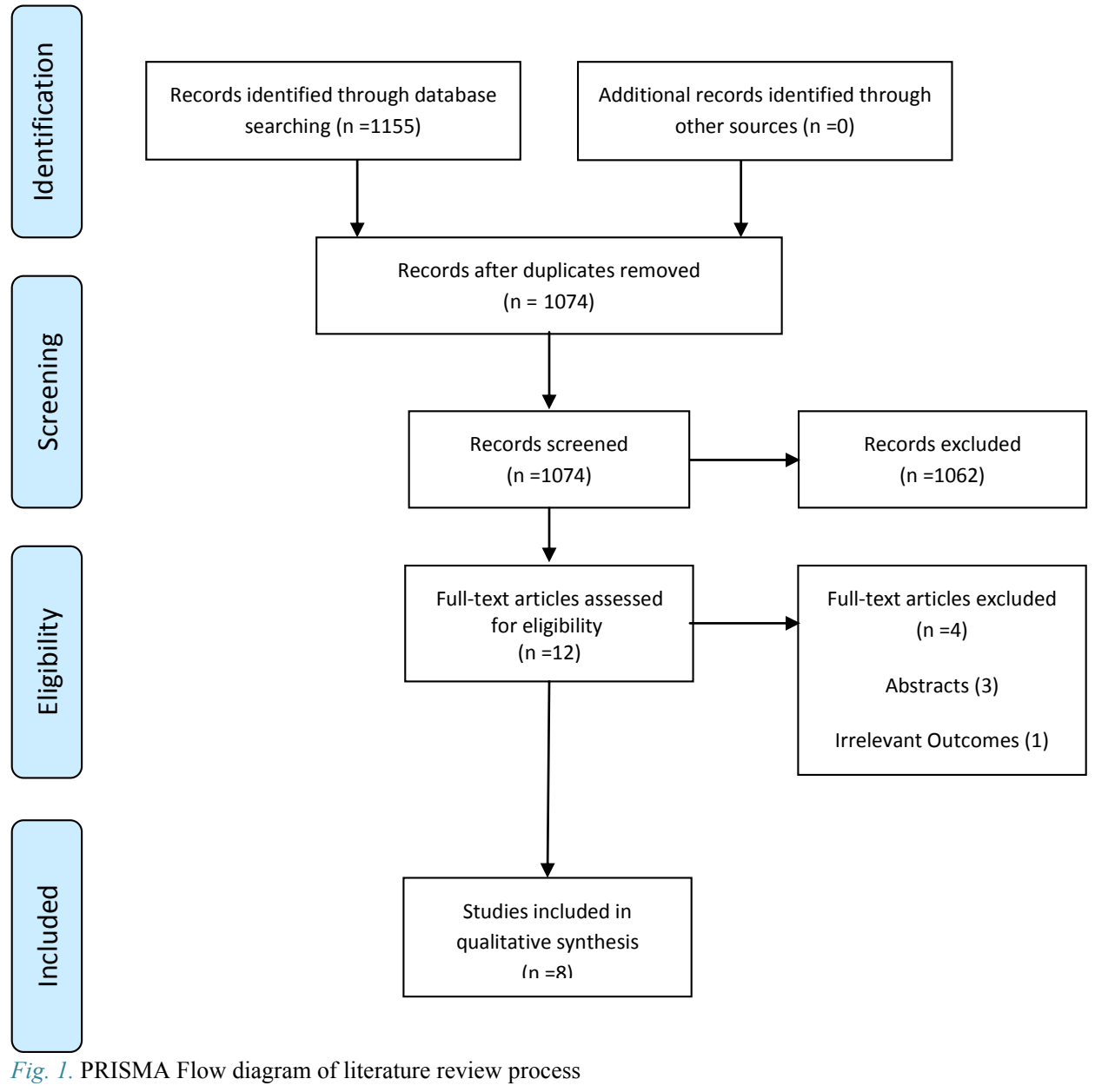




\begin{tabular}{|c|c|c|c|c|c|c|c|c|c|}
\hline $\begin{array}{l}\text { Author, Year, Coun- } \\
\text { try }\end{array}$ & $\begin{array}{l}\text { Type of } \\
\text { economic } \\
\text { evaluation }\end{array}$ & Perspective & Time horizon & $\begin{array}{c}\text { Costs } \\
\text { (types of costs, } \\
\text { sources of cost data) }\end{array}$ & $\begin{array}{l}\text { Type of } \\
\text { effects }\end{array}$ & $\begin{array}{l}\text { Discount rates } \\
\text { (costs, effects) }\end{array}$ & $\begin{array}{l}\text { Type of sensitivity } \\
\text { analysis }\end{array}$ & $\begin{array}{l}\text { Willing-To-Pay } \\
\text { Threshold }\end{array}$ & $\begin{array}{l}\text { Industry } \\
\text { funding }\end{array}$ \\
\hline $\begin{array}{l}\text { Alvarado et al, 2013, } \\
\text { USA (20) }\end{array}$ & $\begin{array}{c}\text { CUA, } \\
\text { Model based, } \\
\text { Markov model }\end{array}$ & societal & 10 years & Reimbursement, published data & QALYs & $3 \%, 3 \%$ & $\begin{array}{l}\text { One-way, two-way, } \\
\text { scenario analysis }\end{array}$ & $\begin{array}{l}\text { US } \$ 75,000 \text { per } \\
\text { QALYs gained }\end{array}$ & NR \\
\hline $\begin{array}{l}\text { Deshmukh et al, } \\
\text { 2017, USA (21) }\end{array}$ & $\begin{array}{c}\text { CUA } \\
\text { Model based, } \\
\text { Markov model }\end{array}$ & $\begin{array}{l}\text { societal and } \\
\text { health care } \\
\text { sector }\end{array}$ & lifetime & $\begin{array}{l}\text { Direct medical care costs, } \\
\text { from the } 2016 \text { Medicare Physi- } \\
\text { cian Fee Schedule and Outpa- } \\
\text { tient Prospective Payment Sys- } \\
\text { tem: indirect care, routine fol- } \\
\text { low-up, and } \\
\text { metastatic care costs from the } \\
\text { literature }\end{array}$ & QALYs & $3 \%, 3 \%$ & $\begin{array}{l}\text { Deterministic (one- } \\
\text { way } \\
\text { and two-way )and } \\
\text { probabilistic }\end{array}$ & $\begin{array}{l}\text { US\$50 } 000 \text { and } \\
\text { US\$100 } 000 \text { per } \\
\text { QALYs gained }\end{array}$ & No \\
\hline $\begin{array}{l}\text { Kamenský et al, } \\
\text { 2019, Czech (26) }\end{array}$ & $\begin{array}{l}\text { CUA, } \\
\text { Model based, } \\
\text { Markov model }\end{array}$ & $\begin{array}{l}\text { healthcare } \\
\text { payer }\end{array}$ & 40 years & direct costs using calculation & QALYs & $3.5 \%, 3.5 \%$ & One way & $\begin{array}{l}1.213 \text { million } \\
\text { CZK }\end{array}$ & NR \\
\hline $\begin{array}{l}\text { Patel et al, 2017, } \\
\text { USA (22) }\end{array}$ & $\begin{array}{l}\text { CUA, } \\
\text { Model based, } \\
\text { Markov model }\end{array}$ & $\begin{array}{l}\text { healthcare } \\
\text { payer }\end{array}$ & lifetime & $\begin{array}{l}\text { direct costs using Medicare re- } \\
\text { imbursement and published } \\
\text { data }\end{array}$ & QALYs, & $3 \%, 3 \%$ & One-way & $\begin{array}{l}\text { US } \$ 50,000 \text { per } \\
\text { QALYs gained }\end{array}$ & $\mathrm{iCAD}$ \\
\hline $\begin{array}{l}\text { Picot et al, 2015, UK } \\
\text { (8) }\end{array}$ & $\begin{array}{c}\text { CUA } \\
\text { Model based, } \\
\text { Markov model }\end{array}$ & $\begin{array}{l}\text { healthcare } \\
\text { payer (NHS } \\
\text { and PSS ) }\end{array}$ & 40 years & Real cost & QALYs & $3.5 \%, 3.5 \%$ & $\begin{array}{l}\text { One-way, scenario } \\
\text { analysis and proba- } \\
\text { bilistic }\end{array}$ & $\begin{array}{l}£ 20,000 \text { and } \\
£ 30,000 \text { per } \\
\text { QALYs }\end{array}$ & No \\
\hline $\begin{array}{l}\text { Shah et al, 2014, } \\
\text { USA (23) }\end{array}$ & $\begin{array}{c}\text { CUA } \\
\text { NR, analyses were based } \\
\text { on } \\
\text { reimbursement models }\end{array}$ & Societal & $\begin{array}{l}\text { NR, assumed } \\
\text { to be } 10 \text { years }\end{array}$ & $\begin{array}{l}\text { Direct and indirect (nonmedi- } \\
\text { cal) costs: Reimbursement }\end{array}$ & QALYs & not reported & No & NR & NR \\
\hline $\begin{array}{l}\text { Vaidya et al, 2017, } \\
\text { UK (24) }\end{array}$ & $\begin{array}{c}\text { CUA, } \\
\text { Model based, } \\
\text { Markov model }\end{array}$ & $\begin{array}{l}\text { National } \\
\text { Health Ser- } \\
\text { vice (NHS) }\end{array}$ & 10 years & $\begin{array}{l}\text { Intervention costs, costs of be- } \\
\text { ing disease free, local recur- } \\
\text { rence and distant recurrence; } \\
\text { expert opinion, NHS reference } \\
\text { costs, published data }\end{array}$ & QALYs & $3.5 \%, 3.5 \%$ & $\begin{array}{l}\text { One-way, probabil- } \\
\text { istic }\end{array}$ & 0.00 & $\begin{array}{c}\text { Carl-Zeiss } \\
\text { Meditec } \\
\text { AG }\end{array}$ \\
\hline $\begin{array}{l}\text { Vaidya et al, 2016, } \\
\text { UK (25) }\end{array}$ & $\begin{array}{c}\text { CUA } \\
\text { Trial based } \\
\text { using } \\
\text { patient-level data from the } \\
\text { TARGIT-A trial }\end{array}$ & $\begin{array}{l}\text { healthcare } \\
\text { payer (NHS } \\
\text { and PSS ) }\end{array}$ & 5 years & $\begin{array}{l}\text { Using resource use and } \\
\text { event data collected prospec- } \\
\text { tively in the trial, published } \\
\text { sources }\end{array}$ & QALYs & $3.5 \%, 3.5 \%$ & deterministic & $\begin{array}{l}£ 20,000- \\
£ 30,000 \text { per } \\
\text { QALYs }\end{array}$ & Carl Zeiss \\
\hline
\end{tabular}

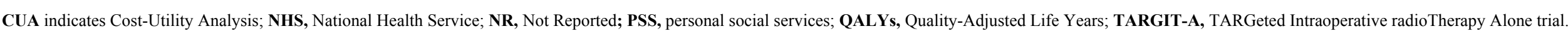


Table 2. Quality of reporting of included studies using CHEERS checklist

\begin{tabular}{|c|c|c|c|c|c|c|c|c|c|}
\hline Section/item & $\begin{array}{c}\text { Item } \\
\text { No }\end{array}$ & $\begin{array}{l}\text { Vaidya et al, } \\
2017(24)\end{array}$ & $\begin{array}{c}\text { Alvarado et } \\
\mathrm{al}, 2013(20)\end{array}$ & $\begin{array}{r}\text { Picot et al, } \\
2015(8) \\
\end{array}$ & $\begin{array}{l}\text { Shah et al, } \\
2014(23)\end{array}$ & $\begin{array}{l}\text { Patel et al, } \\
2017(22) \\
\end{array}$ & $\begin{array}{l}\text { Kamenský et } \\
\mathrm{al}, 2019(26)\end{array}$ & $\begin{array}{l}\text { Vaidya et al, } \\
2016(25)\end{array}$ & $\begin{array}{l}\text { Deshmukh et } \\
\text { al, 2017 (21) }\end{array}$ \\
\hline \multicolumn{10}{|l|}{ Title and abstract } \\
\hline Title & 1 & Part & Part & Part & Part & Yes & Part & Yes & Yes \\
\hline Abstract & 2 & Part & Part & Yes & Part & Part & Part & Part & Yes \\
\hline \multicolumn{10}{|l|}{ Introduction } \\
\hline Background and objectives & 3 & Yes & Yes & Yes & Yes & Yes & Part & Yes & Yes \\
\hline \multicolumn{10}{|l|}{ Methods } \\
\hline Target population and subgroups & 4 & Yes & Yes & Yes & Part & Yes & No & Yes & Yes \\
\hline Setting and location & 5 & Yes & Part & Yes & No & Part & No & Part & Yes \\
\hline Study perspective & 6 & Yes & Yes & Yes & Part & Yes & Part & Yes & Yes \\
\hline Comparators & 7 & Yes & Yes & Yes & Part & Yes & Part & Yes & Yes \\
\hline Time horizon & 8 & Yes & Part & Yes & No & Part & Part & Yes & Yes \\
\hline Discount rate & 9 & Yes & Yes & Yes & No & Yes & Part & Yes & Yes \\
\hline Choice of health outcomes & 10 & Yes & Part & Yes & Part & Yes & Part & Yes & Yes \\
\hline \multirow[t]{2}{*}{ Measurement of effectiveness } & $11 \mathrm{a}$ & NA & NA & NA & Part & NA & NA & Yes & NA \\
\hline & $11 \mathrm{~b}$ & Yes & Part & Yes & NA & Yes & Part & NA & Yes \\
\hline Measurement and valuation of preference based outcomes & 12 & Yes & Part & Yes & Part & $\mathrm{Na}$ & Part & Yes & Yes \\
\hline \multirow[t]{2}{*}{ Estimating resources and costs } & $13 \mathrm{a}$ & NA & NA & NA & Part & NA & NA & Yes & NA \\
\hline & $13 \mathrm{~b}$ & Yes & Part & Yes & NA & Part & No & NA & Yes \\
\hline Currency, price date, and conversion & 14 & Part & Part & Part & No & Part & No & Part & Yes \\
\hline Choice of model & 15 & Part & Part & Yes & No & Part & Part & Part & Yes \\
\hline Assumptions & 16 & Yes & Yes & Yes & Part & Yes & No & Part & Yes \\
\hline Analytical methods & 17 & Yes & Yes & Yes & No & Yes & No & Yes & Yes \\
\hline \multicolumn{10}{|l|}{ Results } \\
\hline Study parameters & 18 & Yes & Yes & Yes & Part & Yes & Part & Part & Yes \\
\hline Incremental costs and outcomes & 19 & Yes & Yes & Yes & Part & Yes & Part & Yes & Yes \\
\hline \multirow{2}{*}{ Characterising uncertainty } & $20 \mathrm{a}$ & NA & NA & NA & No & NA & NA & No & NA \\
\hline & $20 \mathrm{~b}$ & Yes & Yes & Yes & NA & Yes & Part & NA & Yes \\
\hline Characterising heterogeneity & 21 & Part & No & Yes & No & No & No & No & Yes \\
\hline Discussion & & & & & & & & & \\
\hline $\begin{array}{l}\text { Study findings, limitations, generalisability, and current knowledge } \\
\text { Other }\end{array}$ & 22 & Yes & Part & Yes & Part & Yes & Part & Yes & Yes \\
\hline Source of funding & 23 & Yes & No & Yes & No & Yes & No & Yes & Yes \\
\hline Conflicts of interest & 24 & Yes & Yes & No & Yes & Yes & No & Yes & No \\
\hline CHEERS Quality Score Out of 24 Points & & 21 & 16.5 & 22 & 8 & 19 & 7 & 19 & 23 \\
\hline
\end{tabular}

CHEERS Consolidated Health Economic Evaluation Reporting Standards, $\boldsymbol{N A}$ not applicable, No not reported, Part partially reported, Yes reported 


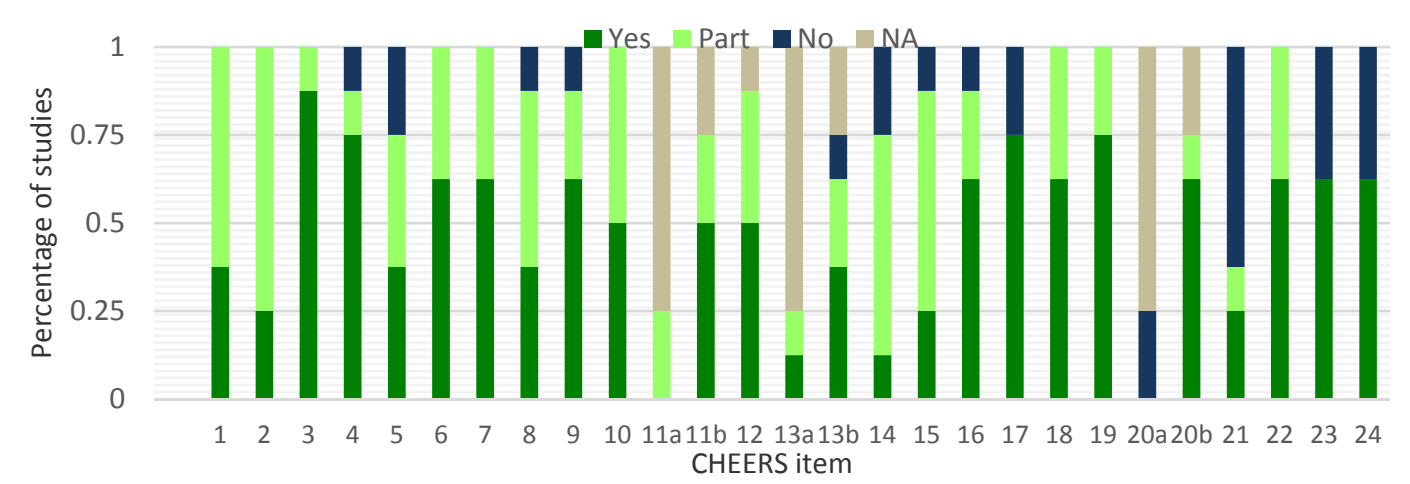

Fig. 2. Quality of reporting of included studies per items of the CHEERS checklist. CHEERS Consolidated Health Economic Evaluation Reporting Standards, NA not applicable, No not reported, Part partially reported, Yes reported

\begin{tabular}{|c|c|c|c|c|c|c|c|c|}
\hline Study & Population & Interventions & Base-case results & $\begin{array}{c}\text { IORT associated } \\
\text { with better } \\
\text { health outcomes? }\end{array}$ & $\begin{array}{c}\text { IORT associated } \\
\text { with more } \\
\text { expensive? }\end{array}$ & $\begin{array}{l}\text { The base case } \\
\text { ICER }\end{array}$ & $\begin{array}{c}\text { Results } \\
\text { of sensitivity analysis }\end{array}$ & Conclusions \\
\hline $\begin{array}{l}\text { Vaidya et al } \\
\text { (24) }\end{array}$ & $\begin{array}{l}\text { A hypothetical cohort of } \\
\text { patients with early breast } \\
\text { cancer based on the pub- } \\
\text { lished health state transition } \\
\text { probability data from the } \\
\text { TARGIT-A } \\
\text { trial }\end{array}$ & $\begin{array}{l}\text { IORT versus } \\
\text { EBRT(15 } \\
\text { fractions) }\end{array}$ & $\begin{array}{c}\text { Costs: IORT } £ 12,455 \\
\text { EBRT£13,280 } \\
\text { QALY: IORT 8.15; EBRT } \\
7.97\end{array}$ & Yes & No & $\begin{array}{l}\text { EBRT domi- } \\
\text { nated }\end{array}$ & $\begin{array}{l}\text { TARGIT IORT was dominant } \\
\text { strategy in all parameter varia- } \\
\text { tions. In the PSA results were } \\
\text { robust over a range model pa- } \\
\text { rameters. Also, TARGIT- } \\
\text { IORT was cost saving in } 98 \% \\
\text { iterations. IORT was cost-ef- } \\
\text { fective at zero thresholds of } \\
\text { WTP. }\end{array}$ & $\begin{array}{l}\text { The TARGIT-IORT is a } \\
\text { dominant strategy over } \\
\text { EBRT, being less costly } \\
\text { and producing higher } \\
\text { QALY. }\end{array}$ \\
\hline $\begin{array}{l}\text { Patel et al } \\
(22)\end{array}$ & $\begin{array}{l}\text { All women ( } 55 \text { year old fe- } \\
\text { males) were initially treated } \\
\text { with breast conserving } \\
\text { surgery (BCS) and fol- } \\
\text { lowed by either IORT or } 6 \\
\text { weeks } \\
\text { EBRT }\end{array}$ & $\begin{array}{c}\text { IORT versus } \\
\text { 6-week WB- } \\
\text { EBRT }\end{array}$ & $\begin{array}{c}\text { Costs: IORT } \$ 53,179 ; 6- \\
\text { week WB-EBRT } \$ 63,828 \\
\text { QALY: IORT 17.86; 6- } \\
\text { week WB-EBRT } 17.06\end{array}$ & Yes & No & $\begin{array}{l}\text { 6-week WB- } \\
\text { EBRT domi- } \\
\text { nated }\end{array}$ & $\begin{array}{l}\text { The findings was most sensi- } \\
\text { tive to the probabilities of re- } \\
\text { current cancer and death for } \\
\text { both IORT and EBRT }\end{array}$ & $\begin{array}{l}\text { IORT was the dominant } \\
\text { strategy(less costly, } \\
\text { more QALYs), and re- } \\
\text { sults in the highest NMB } \\
\text { (\$839,815 vs. \$789,092) } \\
\text { and a lower cost per } \\
\text { QALY compared to } \\
\text { EBRT (\$3039 vs. } \\
\$ 3741) \text {. }\end{array}$ \\
\hline
\end{tabular}

CUA indicates Cost-Utility Analysis; EBRT, External Beam Radiation Therapy; ELIOT, Electron Intraoperative Radiotherapy Trial; ER+, Estrogen Receptor Positive; ICER, Incremental Cost-Effectiveness Ratio; IORT, Intraoperative Radiation

Therapy; NMB, Net Monetary Benefit; QALYs, Quality-Adjusted Life Years; TARGIT-A, TARGeted Intraoperative radioTherapy Alone trial; WB, Whole Breast

$6 \quad$ http://mjiri.iums.ac.ir

Med J Islam Repub Iran. 2020 (10 Dec); 34:167. 


\begin{tabular}{|c|c|c|c|c|c|c|c|c|}
\hline $\begin{array}{l}\text { Study } \\
\end{array}$ & Population & Interventions & Base-case results & $\begin{array}{l}\text { IORT associ- } \\
\text { ated with bet- } \\
\text { ter } \\
\text { health out- } \\
\text { comes? }\end{array}$ & $\begin{array}{l}\text { IORT asso- } \\
\text { ciated with } \\
\text { more } \\
\text { expensive? }\end{array}$ & The base case ICER & $\begin{array}{c}\text { Results } \\
\text { of sensitivity analysis }\end{array}$ & Conclusions \\
\hline $\begin{array}{l}\text { Kamenský } \\
\text { et al (26) }\end{array}$ & $\begin{array}{l}\text { based on the TARGIT-A } \\
\text { pragmatic randomized } \\
\text { controlled trial }\end{array}$ & $\begin{array}{l}\text { IORT versus } \\
\text { 6-week WB- } \\
\text { EBRT }\end{array}$ & $\begin{array}{l}\text { Costs: IORT CZK 47,585; 6-week } \\
\text { WB-EBRT CZK 62,784 } \\
\text { QALY: IORT 14.269; 6-week WB- } \\
\text { EBRT 14.553 }\end{array}$ & No & No & $\begin{array}{c}53,483 \\
\text { (CZK saved/QALY } \\
\text { lost) }\end{array}$ & $\begin{array}{l}\text { The results of the sensitivity } \\
\text { analysis are consistent with } \\
\text { the results of the baseline sce- } \\
\text { nario. }\end{array}$ & $\begin{array}{l}\text { The ICER ratio of the } \\
\text { IORT was below the } \\
\text { threshold, and therefore } \\
\text { did } \\
\text { not meet the criteria for } \\
\text { adoption of the new tech- } \\
\text { nology }\end{array}$ \\
\hline $\begin{array}{l}\text { Alvarado } \\
\text { et al (20) }\end{array}$ & $\begin{array}{l}\text { Women aged } \geq 55 \text { years } \\
\text { with early breast } \\
\text { cancer defined as stage I- } \\
\text { IIA ER+ included in } \\
\text { TARGIT-A trial }\end{array}$ & $\begin{array}{l}\text { IORT versus } \\
\text { 6-week WB- } \\
\text { EBRT }\end{array}$ & $\begin{array}{l}\text { Costs: IORT } \$ 28,879 ; 6 \text {-week WB- } \\
\text { EBRT } \$ 34,070 \\
\text { QALY: IORT 7.66020; 6-week WB- } \\
\text { EBRT } 7.65994\end{array}$ & Yes & No & $\begin{array}{c}\text { 6-week WB-EBRT } \\
\text { dominated }\end{array}$ & $\begin{array}{l}\text { The model was most sensitive } \\
\text { to health state utilities and lo- } \\
\text { cal and distant recurrence } \\
\text { rates. }\end{array}$ & $\begin{array}{l}\text { IORT dominates WB- } \\
\text { EBRT. The ICER showed } \\
\text { that IORT dominated WB- } \\
\text { EBRT by being both } \\
\text { cheaper and more clini- } \\
\text { cally effective. IORT is the } \\
\text { preferred strategy except } \\
\text { when the } 10 \text {-year LRR of } \\
\text { IORT is high ([3.11\%) or } \\
\text { the proportion receiving } \\
\text { EBRT after IORT is high } \\
(24 \%) \text {. }\end{array}$ \\
\hline $\begin{array}{l}\text { Deshmukh } \\
\text { et al (21) }\end{array}$ & $\begin{array}{l}\text { Women with an age range } \\
\text { of } 45 \text { to } 75 \text { years treated } \\
\text { with BCS for stage I/II } \\
\text { breast cancer }\end{array}$ & $\begin{array}{l}\text { IORT versus } \\
\text { 3-week WB- } \\
\text { EBRT versus } \\
\text { 6-week WB- } \\
\text { EBRT }\end{array}$ & $\begin{array}{l}\text { Societal perspective: Costs: IORT; } \\
\$ 42,410 ; 3 \text {-week WB-EBRT } \$ 47,486 \text {; } \\
\text { 6-week WB-EBRT } \$ 50,981 \\
\text { QALY: IORT 12.1764; 3-week WB- } \\
\text { EBRT 12.4745; } \\
\text { 6-week WB-EBRT 12.2929 } \\
\text { Health care sector perspective: } \\
\text { Costs: IORT; } \$ 42,345 ; 3 \text {-week WB- } \\
\text { EBRT } \$ 46,783 ; \\
\text { 6-week WB-EBRT } \$ 49,433 \\
\text { QALY: IORT 12.1764; 3-week WB- } \\
\text { EBRT 12.4745; } \\
\text { 6-week WB-EBRT } 12.2929\end{array}$ & No & No & $\begin{array}{c}\text { 6-week WB-EBRT } \\
\text { dominated. } \\
\text { ICER for 3-week } \\
\text { WB-EBRT versus } \\
\text { IORT: } \\
\text { Societal : } \$ 17,024 \\
\text { per QALY } \\
\text { Health care sector: } \\
\$ 14,886 \text { per QALY }\end{array}$ & $\begin{array}{l}\text { The ICER was most sensitive } \\
\text { to the probability of metastasis } \\
\text { after treatment and treatment } \\
\text { cost of 3-week WB-EBRT and } \\
\text { IORT }\end{array}$ & $\begin{array}{l}\text { 3-week WB-EBRT was } \\
\text { cost-effective compared } \\
\text { with 6-week WB-EBRT } \\
\text { and IORT. The probability } \\
\text { that 3-week WB-EBRT } \\
\text { was cost-effective was } \\
75 \% \text { and } 80 \% \text { at willing- } \\
\text { ness-to-pay thresholds of } \\
\$ 50000 \text { per QALY and } \\
\$ 100000 \text { per QALY, re- } \\
\text { spectively. }\end{array}$ \\
\hline
\end{tabular}




\begin{tabular}{|c|c|c|c|c|c|c|c|c|}
\hline Study & Population & Interventions & Base-case results & $\begin{array}{l}\text { IORT associ- } \\
\text { ated with bet- } \\
\text { ter } \\
\text { health out- } \\
\text { comes? } \\
\end{array}$ & $\begin{array}{l}\text { IORT asso- } \\
\text { ciated with } \\
\text { more } \\
\text { expensive? }\end{array}$ & $\begin{array}{l}\text { The base case } \\
\text { ICER }\end{array}$ & $\begin{array}{c}\text { Results } \\
\text { of sensitivity analysis }\end{array}$ & Conclusions \\
\hline $\begin{array}{l}\text { Picot et al } \\
(8)\end{array}$ & $\begin{array}{l}\text { Patient population in the } \\
\text { pre-pathology stratum of } \\
\text { the TARGIT-A trial. }\end{array}$ & $\begin{array}{l}\text { IORT versus } \\
\text { WB-EBRT } \\
\text { (15 fractions) }\end{array}$ & 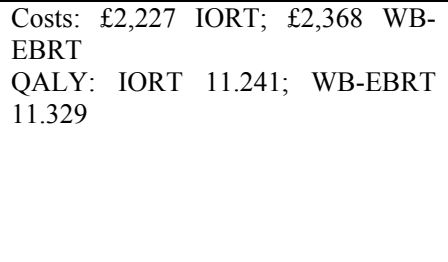 & No & No & $\begin{array}{l}\text { 1596(£ saved/ } \\
\text { QALY lost) }\end{array}$ & $\begin{array}{l}\text { The model were most sensitive } \\
\text { to the probability of any other re- } \\
\text { currence for WB-EBRT and } \\
\text { IORT, the beta coefficient for } \\
\text { the time to local recurrence } \\
\text { (IORT) and the probability of } \\
\text { death from breast cancer } \\
\text { (IORT). }\end{array}$ & $\begin{array}{l}\text { IORT was not cost-effec- } \\
\text { tive compared with WB- } \\
\text { EBRT at the WTP thresh- } \\
\text { old of } £ 20,000 \text { per QALY } \\
\text { as the cost saved per } \\
\text { QALY lost was less than } \\
£ 20,000 \text {. }\end{array}$ \\
\hline $\begin{array}{l}\text { Shah et al } \\
\text { (23) }\end{array}$ & $\begin{array}{l}\text { TARGIT-A trial: women } \\
\text { with } \quad \text { early- } \\
\text { breast cancer }, \geq 45 \text { years }\end{array}$ & $\begin{array}{l}\text { IORT versus } \\
\text { 6-weekWB- } \\
\text { EBRT }\end{array}$ & $\begin{array}{l}\text { Reimbursement costs ranges: IORT } \\
\$ 3,094 \text { to } \$ 10,179 ; \text { WB-EBRT } \\
\$ 11,726 \text { to } \$ 13,743 \\
\text { QALY: IORT } 9.04 \text {; WB-EBRT } 9.08\end{array}$ & No & Yes & $\begin{array}{l}\$ 89,234 \text { to } \\
\$ 108,735 \text { cost per } \\
\text { QALY for WB- } \\
\text { EBRT versus } \\
\text { IORT depending } \\
\text { on the difference } \\
\text { in whole-breast } \\
\text { irradiation rates }\end{array}$ & - & $\begin{array}{l}\text { INTRABEAM IORT was } \\
\text { a cost-saving option com- } \\
\text { pared with WB-EBRT, but } \\
\text { when other medical and } \\
\text { non-medical costs were in- } \\
\text { cluded, WB-EBRT } \\
\text { showed a cost-effective op- } \\
\text { tion compared with } \\
\text { IORT. }\end{array}$ \\
\hline $\begin{array}{l}\text { Vaidya et } \\
\text { al (25) }\end{array}$ & $\begin{array}{l}817 \text { patients randomized } \\
\text { in the 'earliest cohort' in } \\
\text { the prepathology stratum } \\
\text { of the TARGIT-A trial }\end{array}$ & $\begin{array}{l}\text { IORT versus } \\
\text { EBRT(15 } \\
\text { fractions) }\end{array}$ & $\begin{array}{l}\text { Incremental costs (£),IORT vs. EBRT: } \\
-685 \\
\text { QALYs gained, IORT vs. EBRT: } \\
0.034\end{array}$ & Yes & No & $\begin{array}{l}\text { 6-week WB- } \\
\text { EBRT dominated }\end{array}$ & $\begin{array}{l}\text { In the deterministic sensitivity } \\
\text { analyses the probability that } \\
\text { IORT was cost-effective at a } \\
\text { maximum willingness to pay for } \\
\text { a QALY of } £ 20,000 \text { and } £ 30,000 \\
\text { was }>0.75 \text { and }>0.80 \text { in every } \\
\text { case respectively. }\end{array}$ & $\begin{array}{l}\text { IORT was less costly than } \\
\text { EBRT, with similar } \\
\text { QALYs, and a positive in- } \\
\text { cremental net monetary } \\
\text { benefit and had a probabil- } \\
\text { ity of }>90 \% \text { of being cost- } \\
\text { effective. }\end{array}$ \\
\hline
\end{tabular}

CUA indicates Cost-Utility Analysis; EBRT, External Beam Radiation Therapy; ELIOT, Electron Intraoperative Radiotherapy Trial; ER+, Estrogen Receptor Positive; ICER, Incremental Cost-Effectiveness Ratio; IORT, Intraoperative Radiation Therapy; NMB, Net Monetary Benefit; QAL Ys, Quality-Adjusted Life Years; TARGIT-A, TARGeted Intraoperative radioTherapy Alone trial; WB, Whole Breast 


\section{Results of Cost-Effectiveness Analysis}

The results of the eight full economic evaluation studies included in this systematic review are summarized in Table 3. In four studies, which took place in the United States and the United Kingdom, IORT was the dominant option (20, $22,24,25)$. In all four studies, this technology was associated with lower costs and higher effectiveness than conventional radiotherapy. These studies were conducted from different perspectives and time horizons. Vaida et al. (25) assessed the cost-utility of TARGIT-IORT during lumpectomy compared with EBRT (15 fractions) in the prepathology stratum of the TARGIT-A trial. The analysis took the UK NHS and personal social services (PSS) perspective and a time horizon of 5 years. The study found that IORT was less costly than EBRT (mean incremental cost -£685) and resulted in slightly more QALYs than EBRT (mean QALYs gained 0.034). The difference in costs between the two groups was statistically significant but the difference in QALYs was not. IORT had a positive incremental net monetary benefit that was borderline statistically significantly different from zero and had a probability of $>90 \%$ of being cost-effective. The study concluded that using IORT routinely instead of EBRT in eligible patients may be a potential budget saving to the NHS (around £8-9.1 million each year). Vaida et al. (24) performed a cost-utility analysis using decision-analytic modeling for the UK setting and National Health Service (NHS healthcare payer's perspective) for a time horizon of 10 years. They found that in the base case analysis, TARGIT-IORT was the dominant strategy over EBRT, yielding higher QALY gain at a lower cost than EBRT. The results were robust to one-way and probabilistic sensitivity analyses. Moreover, based on probabilistic analysis, TARGIT-IORT had a $98 \%$ chance of being cost-effective at zero WTP. Alvarado et al. (20). reported a full economic evaluation study based on the US health-care system by developing a Markov decision model to assess the cost-effectiveness of IORT(INTRABEAM) compared with WB-EBRT, based on the trial results of the TARGITA. The analysis was performed over a 10-year time horizon and from a societal perspective. The study concluded that single-dose IORT was the dominant, more cost-effective strategy that provides greater QALYs at a decreased cost compared with 6-week WB-EBRT. The model was most sensitive to health state utilities and local and distant recurrence rates. IORT was always preferred, and in most cases, the dominant strategy across all sensitivity analyses. In all of the probability and rate sensitivity analyses, the ICER for WB-EBRT was significantly greater than the society's willingness-to-pay of $\$ 75,000 / \mathrm{QALY}$. In addition, the scenario analysis showed that IORT was the dominant strategy compared with a 3-week accelerated WB-EBRT schedule of 16 fractions in terms of both QALYs and life expectancy. In this study, a probabilistic sensitivity analysis (PSA) was not conducted. Patel et al. (22). used a Markov model to assess the cost-effectiveness of IORT versus a 6-week WB-EBRT in treating early-stage (stage I-IIA/IIB) breast cancer over the life of the patients for the USA setting from the healthcare payer and found that IORT was the dominant (less costly with greater QALYs) versus EBRT and at a willingness to pay of
$\$ 50,000$ for each additional QALY, the net monetary benefit demonstrated that IORT was the most cost-effective option. The model used in their study was sensitive to the probabilities of recurrent cancer and death for both IORT and EBRT. The study concluded that IORT was the more cost-effective option (lower cost with improved QALYs) for use in patients with early-stage ER+ breast cancer.

In three studies, Shah et al., Kamensky et al., and Picot et al., the dominant option was conventional radiotherapy (8, $23,26)$. In these studies, IORT also was associated with fewer costs, but its effectiveness was lower than that of conventional radiotherapy. Shah et al. (23) carried out an economic evaluation based on TARGIT-A and the Electron Intraoperative Radiotherapy (ELIOT) trial and a societal perspective, including both direct and indirect costs, for a time horizon of 10 years in the USA. They found that EBRT was a more cost-effective treatment compared to IORT. In the study, the costs per QALY for WB-EBRT compared with INTRABEAM IORT ranged from $\$ 89,234$ to $\$ 108,735$ depending on the difference in whole-breast irradiation rates. The study concluded that IORT is a potential cost-saving in the management of early-stage breast cancer But, WBI represents a cost-effective option and remains the standard of care. Using a Markov model, Kamensky et al. (26) assessed cost-utility from the Czech healthcare system perspective. The study found the ICER value was CZK 53483 saved per 1 QALY lost. The results of the sensitivity analysis were consistent with the results of the baseline scenario. Then, in the basic scenario, IORT was less expensive but less effective than EBRT. The ICER of the IORT versus EBRT was below the threshold of 1.213 million CZK and therefore, in this study IORT was found not to be cost-effective for patients with early breast cancer. Picot et al. in their study (8) assessed the cost-effectiveness of IORT compared with WB-EBRT for early breast cancer from the NHS perspective and a lifetime (40-year) horizon in the UK. The study found IORT to be less costly but also less effective than WB-EBRT. The base-case ICER to replace WB-EBRT with intraoperative radiation therapy was $£ 1596$ saved per QALY lost. Therefore, IORT was not costeffective at a willingness-to-pay (WTP) threshold of $£ 20,000$ per QALY. The PSA indicated that WB-EBRT at the $£ 20,000$ and $£ 30,000$ WTP thresholds and IORT at thresholds of around $£ 5000$ per QALY or less has a greater probability than each other of being cost-effective

In the study of Deshmukh et al. (21), which was conducted with two social and health care perspectives, 3-week radiotherapy was the dominant option as compared to 6week radiotherapy IORT. In this study, 6-week radiotherapy was a dominated option and IORT was less costly and less effective than 3-week radiotherapy. In the last four studies, IORT was not cost-effective on the basis of the willingness to pay thresholds, but these studies pointed to the potential for the cost-effectiveness of $\operatorname{IORT}(8,21,23$, 26). In these studies, the money saved per QALY lost due to the replacement of IORT with conventional radiotherapy was used. Similar results were obtained in the study of Kamensky et al. and Picot et al., which used the same Markov models $(8,26)$. Finally, there was also heterogeneity in terms of the thresholds of willingness to pay, the structure 
of models and their assumptions among the included studies, even in studies conducted in the United States or the United Kingdom.

\section{Drivers of Cost-Effectiveness}

One-way sensitivity analyses were reported in 7 out of 8 included studies. Yet, numerous studies did not perform one-way sensitivity analyses on all model parameters or only conducted one-way sensitivity or scenario analyses on a few input parameters. Among the eight included studies, the model was most sensitive to probabilities of recurrent cancer and death for both IORT and EBRT (22), health state utilities and local and distant recurrence rates (20), the probability of metastasis after treatment, and treatment cost of HF-WBI and IORT (21), and the probability of any other recurrence assumed for WB-EBRT and INTRABEAM, the beta coefficient for the time to local recurrence (INTRABEAM) and the probability of death from breast cancer (INTRABEAM) (8). In the remaining studies, model outputs were robust to one-way and probabilistic sensitivity analyses.

\section{Discussion}

In this study, we reviewed eight full economic evaluation IORT in comparison with conventional radiotherapy in adjunct management of early breast cancer. The quality of the studies based on the average reporting quality score of the 8 articles reviewed by the Cheers checklist was moderate (17/24). The cost-effectiveness results of the IORT showed that this technology is located in two areas in the cost-effectiveness plane (more effective, less costly, and less effective and less costly). However, in all studies, IORT reduced costs in comparison with conventional and even hypofractionation WB-EBRT, but this reduction was trivial in the Picot study. On the other hand, in three studies, QALYs were improved with IORT compared to the WBI. In other studies, QALYs were reduced for IORT compared to the WBI. In terms of cost-effectiveness results, we can say that the current evidence is scattered, and the number of studies conducted is low. In general, by reviewing eight economic assessments, there can be no definitive answer to the cost-effectiveness of IORT, but this evidence suggests that IORT can be a cost-effective alternative to early breast cancer treatment by reducing therapeutic costs. So that the cost of IORT in all studies was lower than the cost of EBRT, and this technology provided some cost savings compared to that. Similar findings are also reported in other studies (27). Including other social costs and travel costs will further add to the cost-effectiveness of the IORT (11). Also, the results of these studies showed that the QALYs differences between IORT and EBRT are low.

In the review study, the results should be interpreted with caution for several reasons. There is heterogeneity in terms of perspectives, time horizons, model assumptions, and the settings of studies. Most studies were conducted in the United States and Canada. Various cost-effectiveness thresholds have been used in different studies. Sources of financing for most studies (5.7 study) were the industry or not mentioned. The evidence shows that industry-funded studies are more likely to report the favorable results of a cost-effectiveness analysis (28).

Sources of effectiveness data for economic models for the majority of included studies are based on efficacy data from TARGIT or Elliot trials that may cause a risk of bias. It seems that, as mentioned in the findings, the effectiveness outcomes in the included studies are influenced by these parameters and can affect the results of the studies (24).

This study has several strengths. First, the present study is one of the first systematic reviews of cost-effectiveness evidence of IORT compared with conventional radiotherapy in early breast cancer. Second, in this study, the quality of the reporting of the studied studies was evaluated, and the strengths and weaknesses of these studies were shown. Identifying the weaknesses of present literature can help to improve future cost-effectiveness analysis studies of these technologies. Third, the present study used the principles of the PRISMA statement for conducting research and reporting.

This study has some limitations. Posters or reports that only had only abstracts and without full text were removed because there was not enough information available to assess reporting quality. Also, studies with non-English full text were not included in the review. Another limitation was that given the fact that these studies were conducted in different countries, it was difficult to compare their ICER results because the thresholds for their willingness to pay were different. Finally, it's worth noting that poor reporting does not necessarily mean poor quality of a study. In our review, we did not assess the methodological quality of studies. In this context, the use of assessment tools such as the Philips checklist (29) can be useful. Finally, we identified cost-effectiveness drivers based on reported findings of sensitivity analysis in the included studies, and we did not conduct additional analysis for the determination of the mentioned drivers.

There is a need for future economic evaluation studies in the field. In future studies, the best practice guidelines for conducting and reporting economic evaluations should be used to ensure that all elements and assumptions in these studies are adequately and transparently reported. Future economic modeling studies should also take into account all the costs and outcomes associated with technology, and from the societal perspective and the right time horizons. In addition, In order to address the uncertainty surrounding the model assumptions, there should be used comprehensive, relevant types of sensitivity analyses to address all principal types of uncertainty including methodological, structural, parameter and patient population-related uncertainty (30).

\section{Conclusion}

We identified eight cost-effectiveness analyses of IORT versus EBRT for early breast cancer published to March 2019. This review shows the need for better reporting and more attention to the model assumptions and structural uncertainty, as well as the more commonly recognized parameter uncertainty by using diverse kinds of sensitivity analyses. The results of this study show that IORT can be a potential cost-saving strategy to the health systems for the adjuvant treatment of early breast cancer if the technology was carried out routinely in eligible patients. However, 
these results should be interpreted with caution because of the heterogeneity of studies and possible publication bias.

\section{Conflict of Interests}

The authors declare that they have no competing interests.

\section{References}

1. Fitzmaurice C, Akinyemiju TF, Al Lami FH, Alam T, Alizadeh-Navaei $\mathrm{R}$, Allen $\mathrm{C}$, et al. Global, regional, and national cancer incidence, mortality, years of life lost, years lived with disability, and disabilityadjusted life-years for 29 cancer groups, 1990 to 2016: a systematic analysis for the global burden of disease study. JAMA Oncol. 2018;4(11):1553-68.

2. Gold HT, Hayes MK. Cost effectiveness of new breast cancer radiotherapy technologies in diverse populations. Breast Cancer Res Treat. 2012;136(1):221-9.

3. Zhang L, Zhou Z, Mei X, Yang Z, Ma J, Chen X, et al. Intraoperative radiotherapy versus whole-breast external beam radiotherapy in earlystage breast cancer: a systematic review and meta-analysis. Medicine. 2015;94(27).

4. Lai HW, Liu LC, Ouyang F, Yao CC, Jan HC, Chang YH, et al. Multicenter study on patient selection for and the oncologic safety of intraoperative radiotherapy (IORT) with the Xoft Axxent ${ }^{\circledR} \mathrm{eBx} \circledast$ System for the management of early stage breast cancer in Taiwan. PloS One. 2017;12(11):e0185876.

5. Group EBCTC. Effect of radiotherapy after breast-conserving surgery on 10-year recurrence and 15-year breast cancer death: meta-analysis of individual patient data for 10801 women in 17 randomised trials. Lancet. 2011;378(9804):1707-16.

6. Group EBCTC. Effects of radiotherapy and of differences in the extent of surgery for early breast cancer on local recurrence and 15-year survival: an overview of the randomised trials. Lancet. 2005;366(9503):2087-106.

7. Mashov R, Tabenkin H. [How family physicians estimate their knowledge and skills in musculoskeletal problems?]. Harefuah. 2011;150(3):242-5, 304.

8. Picot J, Copley V, Colquitt JL, Kalita N, Hartwell D, Bryant J. The INTRABEAM(R) Photon Radiotherapy System for the adjuvant treatment of early breast cancer: a systematic review and economic evaluation. Health Technol Assess (Winchester, England). 2015;19(69):1-190.

9. Vaidya JS, Wenz F, Bulsara M, Tobias JS, Joseph DJ, Keshtgar M, et al. Risk-adapted targeted intraoperative radiotherapy versus wholebreast radiotherapy for breast cancer: 5-year results for local control and overall survival from the TARGIT-A randomised trial. Lancet. 2014;383(9917):603-13

10. Vaidya JS, Bulsara M, Wenz F, Joseph D, Saunders C, Massarut S, et al. Pride, prejudice, or science: Attitudes towards the results of the TARGIT-A trial of targeted intraoperative radiation therapy for breast cancer. Int J Radiat Oncol Biol Phys. 2015;92(3):491-7.

11. Bargallo-Rocha JE, Soto-Perez-de-Celis E, Picó-Guzmán FJ, Quintero-Rodríguez CE, Almog D, Santiago-Concha G, et al. The impact of the use of intraoperative radiotherapy on costs, travel time and distance for women with breast cancer in the Mexico City Metropolitan Area. J Surg Oncol. 2017;116(6):683-9.

12. Husereau D, Drummond M, Petrou S, Carswell C, Moher D, Greenberg D, et al. Consolidated health economic evaluation reporting standards (CHEERS) statement. Cost Eff Resour Alloc. 2013;11(1):6.

13. Husereau D, Drummond M, Petrou S, Carswell C, Moher D, Greenberg D, et al. Consolidated health economic evaluation reporting standards (CHEERS) - explanation and elaboration: a report of the ISPOR health economic evaluation publication guidelines good reporting practices task force. Value Health. 2013;16(2):231-50.

14. Hiligsmann M, Evers SM, Sedrine WB, Kanis JA, Ramaekers B, Reginster JY, et al. A systematic review of cost-effectiveness analyses of drugs for postmenopausal osteoporosis. Pharmacoeconomics. 2015;33(3):205-24.

15. Moher D, Liberati A, Tetzlaff J, Altman DG. Preferred reporting items for systematic reviews and meta-analyses: the PRISMA statement. Ann Intern Med. 2009;151(4):264-9.

16. Soto-Molina H, Juarez YE, Sanchez-Trejo K. Economic evaluation of the mobile electron accelerator for intraoperative radiotherapy in patients with breast cancer. Value Health. 2018;21:S33-S.

17. Vaidya A, Vaidya P, Both B. Cost-utility analysis (cua) of targeted intraoperative radiotherapy alone (targit-a) in the brazilian early breast cancer patients. Value Health. 2017;20(9):A854-A.

18. Vaidya A, Vaidya P, Both B, Brew-Graves C, Vaidya J. Cost Effectiveness Analysis of Targeted Intraoperative Radiotherapy Alone (TARGIT-A) in Early Breast Cancer Patients. Value Health. 2014;17(7):A640.

19. Esserman LJ, Alvarado MD, Howe RJ, Mohan AJ, Harrison B, Park $\mathrm{C}$, et al. Application of a decision analytic framework for adoption of clinical trial results: are the data regarding TARGIT-A IORT ready for prime time? Breast Cancer Res Treat. 2014;144(2):371-8.

20. Alvarado MD, Mohan AJ, Esserman LJ, Park CC, Harrison BL, Howe $\mathrm{RJ}$, et al. Cost-Effectiveness Analysis of Intraoperative Radiation Therapy for Early-Stage Breast Cancer. Ann Surg Oncol 2013;20(9):2873-80.

21. Deshmukh AA, Shirvani SM, Lal L, Swint JM, Cantor SB, Smith BD, et al. Cost-effectiveness Analysis Comparing Conventional, Hypofractionated, and Intraoperative Radiotherapy for Early-Stage Breast Cancer. J. Natl. Cancer Inst. 2017;109(11).

22. Patel R, Ivanov O, Voigt J. Lifetime cost-effectiveness analysis of intraoperative radiation therapy versus external beam radiation therapy for early stage breast cancer. Cost Eff Resour Alloc. 2017;15:22.

23. Shah C, Badiyan S, Khwaja S, Shah H, Chitalia A, Nanayati A, et al. Evaluating Radiotherapy Options in Breast Cancer: Does Intraoperative Radiotherapy Represent the Most Cost-Efficacious Option? Clin Breast Cancer. 2014;14(2):141-6.

24. Vaidya A, Vaidya P, Both B, Brew-Graves C, Bulsara M, Vaidya JS. Health economics of targeted intraoperative radiotherapy (TARGITIORT) for early breast cancer: a cost-effectiveness analysis in the United Kingdom. BMJ Open. 2017;7(8):e014944.

25. Vaidya JS, Wenz F, Bulsara M, Tobias JS, Joseph DJ, Saunders C, et al. An international randomised controlled trial to compare TARGeted Intraoperative radioTherapy (TARGIT) with conventional postoperative radiotherapy after breast-conserving surgery for women with early-stage breast cancer (the TARGIT-A trial). Health Technol Assess. 2016;20(73):1-188.

26. Kamensky V, Donin G, Burianova V, Gajdos O, Rogalewicz V, Kubatova I, et al. Cost-Effectiveness Analysis of Intrabeam System Introduction to the Czech Healthcare System Environment. In: Lhotska L, Sukupova L, Lackovic I, Ibbott GS, editors. World Congress on Medical Physics and Biomedical Engineering 2018, Vol 2. IFMBE Proceedings. 682019. p. 107-12.

27. Intrabeam radiotherapy system for adjuvant treatment of early breast cancer The National Institute for Health and Care Excellence: The National Institute for Health and Care Excellence; 2018. Available from: https://www.nice.org.uk/guidance/ta501/resources/intrabeamradiotherapy-system-for-adjuvant-treatment-of-early-breast-cancerpdf-82606714503109.

28. Bell CM, Urbach DR, Ray JG, Bayoumi A, Rosen AB, Greenberg D, et al. Bias in published cost effectiveness studies: systematic review. BMJ. 2006;332(7543):699-703.

29. Philips Z, Bojke L, Sculpher M, Claxton K, Golder S. Good practice guidelines for decision-analytic modelling in health technology assessment. Pharmacoeconomics. 2006;24(4):355-71.

30. Moloney E, O'Connor J, Craig D, Robalino S, Chrysos A, Javanbakht $\mathrm{M}$, et al. Systematic Review of Economic Models Used to Compare Techniques for Detecting Peripheral Arterial Disease. PharmacoEconomics-open. 2019;3(1):21-30. 
Appendix 1. Search strategies and results for selected databases

Database: PubMed

Date conducted: June 25, 2020

Search strategy:

(((("breast neoplasms"[MeSH Terms] OR ("breast"[All Fields] AND "neoplasms"[All Fields]) OR "breast neoplasms"[All Fields] OR ("breast"[All

Fields] AND "cancer"[All Fields]) OR "breast cancer"[All Fields]) OR ("breast neoplasms"[MeSH Terms] OR ("breast"[All Fields] AND "neoplasms"[All Fields]) OR "breast neoplasms"[All Fields] OR ("breast"[All Fields] AND "tumour"[All Fields]) OR "breast tumour"[All Fields])) OR ("breast neoplasms"[MeSH Terms] OR ("breast"[All Fields] AND "neoplasms"[All Fields]) OR "breast neoplasms"[All Fields] OR ("breast"[All Fields] AND "carcinoma"[All Fields]) OR "breast carcinoma"[All Fields])) OR ("breast neoplasms"[MeSH Terms] OR ("breast"[All Fields] AND "neoplasms"[All Fields]) OR "breast neoplasms"[All Fields])) OR "breast neoplasms"[MeSH Terms]) AND (((IORT[All Fields] OR INTRABEAM[All Fields]) OR ELIOT[All Fields]) OR TARGIT[All Fields]) OR (intraoperative[All Fields] AND ("radiotherapy"[Subheading] OR "radiotherapy"[All Fields] OR "radiotherapy"[MeSH Terms])))

Database: Web of Science

Date conducted: June 25, 2020

Search strategy:

TS=((breast tumour OR breast carcinoma OR breast neoplasms OR breast cancer OR "Breast Neoplasms") AND (intraoperative radiotherapy OR TARGIT OR ELIOT OR INTRABEAM)AND (cost* OR economic* OR "economic evaluation" OR "cost effectiveness" OR "cost-effectiveness" OR "cost utility" OR "cost -utility"))

Timespan: All years. Indexes: SCI-EXPANDED, SSCI, A\&HCI, ESCI.

Database: Cochrane Central Register of Controlled Trials

Date conducted: June 25, 2020

Search strategy:

\begin{tabular}{cc}
\hline Search & Query \\
\hline$\# 1$ & MeSH descriptor: [Breast Neoplasms] explode all trees \\
$\# 2$ & MeSH descriptor: [Radiotherapy, Adjuvant] explode all trees \\
$\# 3$ & $\# 1$ and \#2 \\
$\# 4$ & MeSH descriptor: [Cost-Benefit Analysis] explode all trees \\
$\# 5$ & $\# 3$ and \#4 \\
\hline
\end{tabular}

Database: Embase

Date conducted: June 25, 2020

Search strategy:

('intraoperative radiotherapy'/exp OR 'intraoperative radiotherapy' OR iort OR targit OR eliot OR 'x ray generator'/exp OR 'x ray generator') AND ('cost benefit analysis'/exp OR 'cost benefit analysis' OR 'cost effectiveness analysis'/exp OR 'cost effectiveness analysis' OR 'cost utility analysis'/exp OR 'cost utility analysis' OR economic*:ab,ti)

Database: Scopus

Date conducted: June 25, 2020

Search strategy:

TITLE-ABS-KEY ("intraoperative radiotherapy" OR "'intraoperative radiation therapy "OR TARGIT OR ELIOT OR INTRABEAM OR IORT) AND TITLE-ABS-KEY ("cost benefit analysis" OR "cost-benefit analysis" OR "cost effectiveness analysis" OR "cost-effectiveness analysis" OR "cost utility analysis" OR "cost-utility analysis" OR cost*OR economic*) 